\title{
Superior Mesenteric Arterial Embolism Associated with an Acute Limb Ischemia: A Case Report and Literature Review
}

\author{
Abdesslam Bouassria ${ }^{1}$, Elbachir Benjelloun', Imane Kamaoui ${ }^{2}$, Hicham Elbouhaddouti ${ }^{1}$, \\ Ouadii Mouaqit ${ }^{1}$, Abdelmalek Ousadden'1, Khalid Mazaz', Khalid Ait Taleb' \\ Laila Sedreddine $^{3}$, Mohammed El Abkari ${ }^{3}$, SidiAdil Ibrahimi ${ }^{3}$, Ihssane Mellouki ${ }^{3}$ \\ ${ }^{1}$ Department of Surgery, School of Medicine and Pharmacy of Fez, Sidi Mohammed Ben Abdellah University, \\ University Hospital HASSAN II, Route de SidiHrazem, Morocco \\ ${ }^{2}$ Department of Radiology, School of Medicine and Pharmacy of Fez, Sidi Mohammed Ben Abdellah University, \\ University Hospital HASSAN II, Route de SidiHrazem, Morocco \\ ${ }^{3}$ Department of Hepatology and Gastroenterology, School of Medicine and Pharmacy of Fez, Sidi Mohammed \\ Ben Abdellah University, University Hospital HASSAN II, Route de SidiHrazem, Morocco \\ Email: ${ }^{*}$ bouassria abdesslam@hotmail.com
}

Received 27 February 2014; revised 31 March 2014; accepted 8 April 2014

Copyright (C) 2014 by authors and Scientific Research Publishing Inc.

This work is licensed under the Creative Commons Attribution International License (CC BY).

http://creativecommons.org/licenses/by/4.0/

(c) (i) Open Access

\begin{abstract}
Introduction: Acute mesenteric ischemia due to an embolism of the superior mesenteric artery (SMA) is associated with a high mortality rate. Over twenty per cent of acute mesenteric embolism cases consist of multiple emboli. Case Presentation: We present a rare case of a 62-year-old man admitted with acute abdominal pain and signs of intestinal occlusion related to an acute mesenteric ischemia due to superior mesenteric arterial embolism. It was associated with a synchronous acute bilateral lower limb ischemia due to embolic arterial occlusion. He underwent an emergency explorative laparotomy with proximal jejunal resection, and the patient made an excellent recovery. As for the acute limb ischemia, it was treated by efficient anticoagulation allowing limb salvage. Conclusion: When treating a superior mesenteric arterial embolism, the possibility of recurrent or multiple arterial thromboembolic events should be considered. A prompt diagnosis, aggressive surgical treatment and intensive care could improve the prognosis.
\end{abstract}

\section{Keywords}

SMA; Limb; Embolism; Ischemia

\footnotetext{
${ }^{*}$ Corresponding author.
}

How to cite this paper: Bouassria, A., et al. (2014) Superior Mesenteric Arterial Embolism Associated with an Acute Limb Ischemia: A Case Report and Literature Review. Open Journal of Gastroenterology, 4, 181-186. 


\section{Introduction}

Acute mesenteric ischemia (AMI) due to an embolism of the superior mesenteric artery (SMA) is a surgical emergency associated with a high mortality rate due to the difficulty in recognizing the condition before bowel infarction occurs. These thromboembolic events consist of multiple emboli in over twenty per cent of cases, which worsens the prognosis. We could find only one case of embolic episode in a lower limb following a SMA embolism that has been previously reported in the literature [1]. We hereby report a new case of AMI due to embolism of the SMA associated with lower limb acute ischemia.

\section{Case Presentation}

A 62-year-old man, chronic smoker, was admitted to the emergency department complaining of history of abdominal pain since 2 days. Our patient was hemodynamically stable with temperature at $37.9^{\circ} \mathrm{C}$, and blood pressure was 120/80 mmHg. Abdominal examination was marked by diffuse abdominal tenderness, and limb examination showed bilateral toes ischemia (Figure 1). Lower limb sensitivity and mobility were preserved, and the popliteal and tibial pulses were found. Laboratory investigations showed that the hemoglobin level was 12.8 $\mathrm{g} / \mathrm{dl}$, and the white blood cell count was significant for a leukocyte count of $19,400 / \mathrm{mm}^{3}$. Computed tomography (CT) scanning showed a mesenteric infarction (Figure 2) due to the occlusion of a branch of the superior mesenteric artery (SMA) by an embolism (Figure 3). CT scan also revealed an ulcerated atherosclerotic plaque in the infra-renal aorta (Figure 4). Therefore, our patient was taken to operating theatre. Laparotomy was done. Intraoperative examination showed a small bowel necrosis starting from the fourth jejunal loop, extending over $70 \mathrm{~cm}$ (Figure 5). A bowel resection was performed (taking away the necrosed jejunal loop), followed by an anastomosis. The pulse of the SMA was present, and a relatively fresh thrombus was observed in the stump of the resected intestines (Figure 6). As for the acute limb ischemia, it was treated by anticoagulation with continued observation. Our patient made an excellent recovery, both in terms of his abdomen that in terms of his lower limbs. He was discharged home in stable conditions 7 days later. Our patient was seen in outpatient clinic at 1 month and 3 months. He had no functional complaints.

\section{Discussion}

Acute mesenteric ischemia refers to the acute intestinal hypo perfusion that can be classified into 4 specific types. The most frequent cause is arterial emboli ( $40 \%$ to $50 \%$ of cases) [2] [3]. A cardiac source as atrial fibrillation is the most common cause of mesenteric emboli. As for our patient, mesenteric emboli was due to an ulcerated aortic thrombus. The second most common cause is acute mesenteric thrombosis (25\% to $30 \%$ of cases) [4] [5]. Non-occlusive mesenteric ischemia is due to a low cardiac output state associated with diffuse mesenteric vasoconstriction. Mesenteric venous thrombosis is the least common cause of mesenteric ischemia (up to $10 \%$ of cases) [6]. The SMA is anatomically susceptible to embolisms because of its large caliber and narrow take-off angle from the aorta. Over 20 percent of acute mesenteric emboli occur with other embolic events [7].

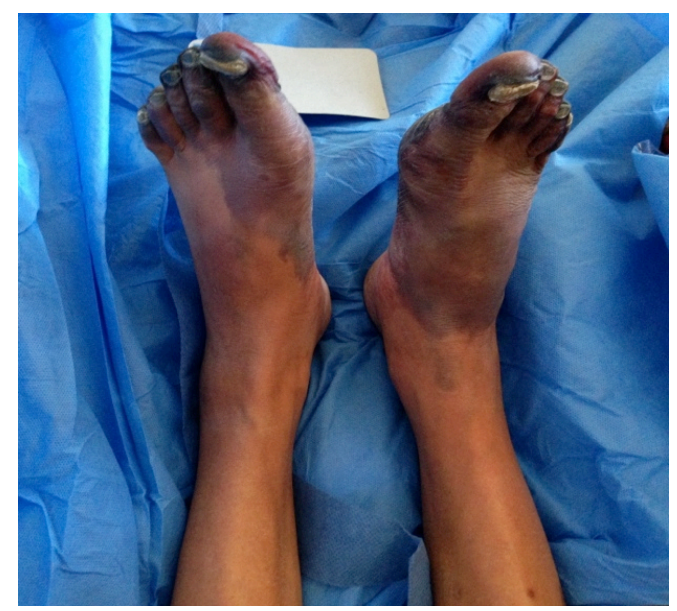

Figure 1. Toes ischemia. 


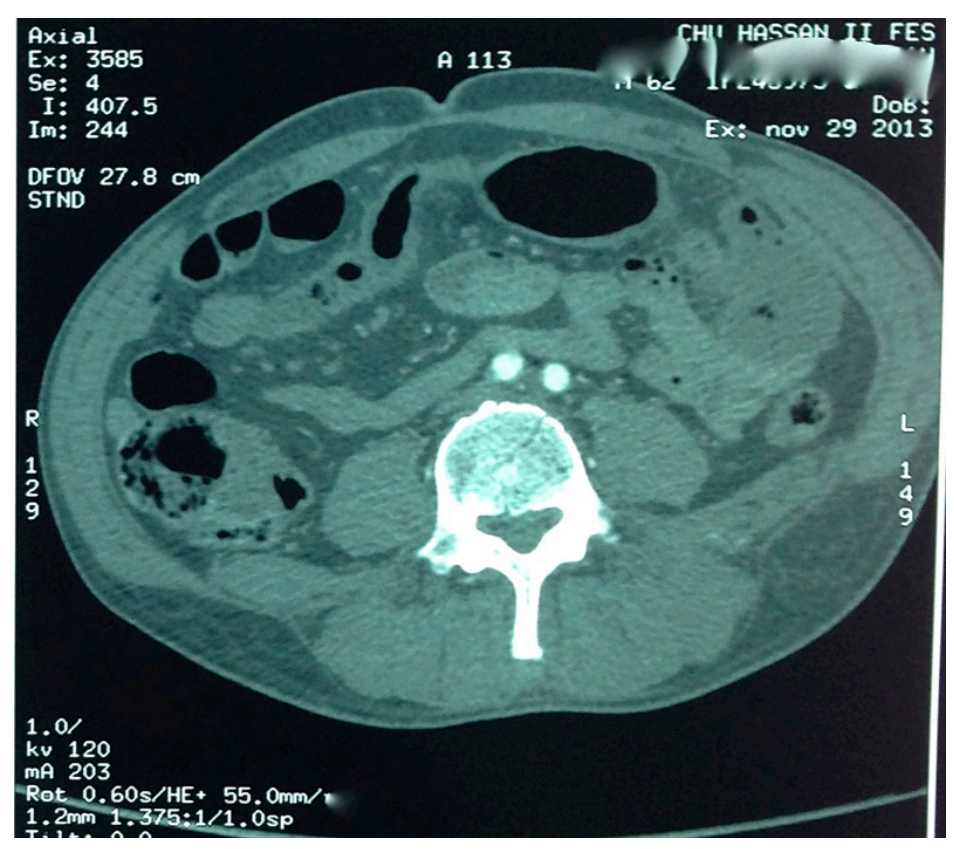

Figure 2. CT scan showing mesenteric infarction.

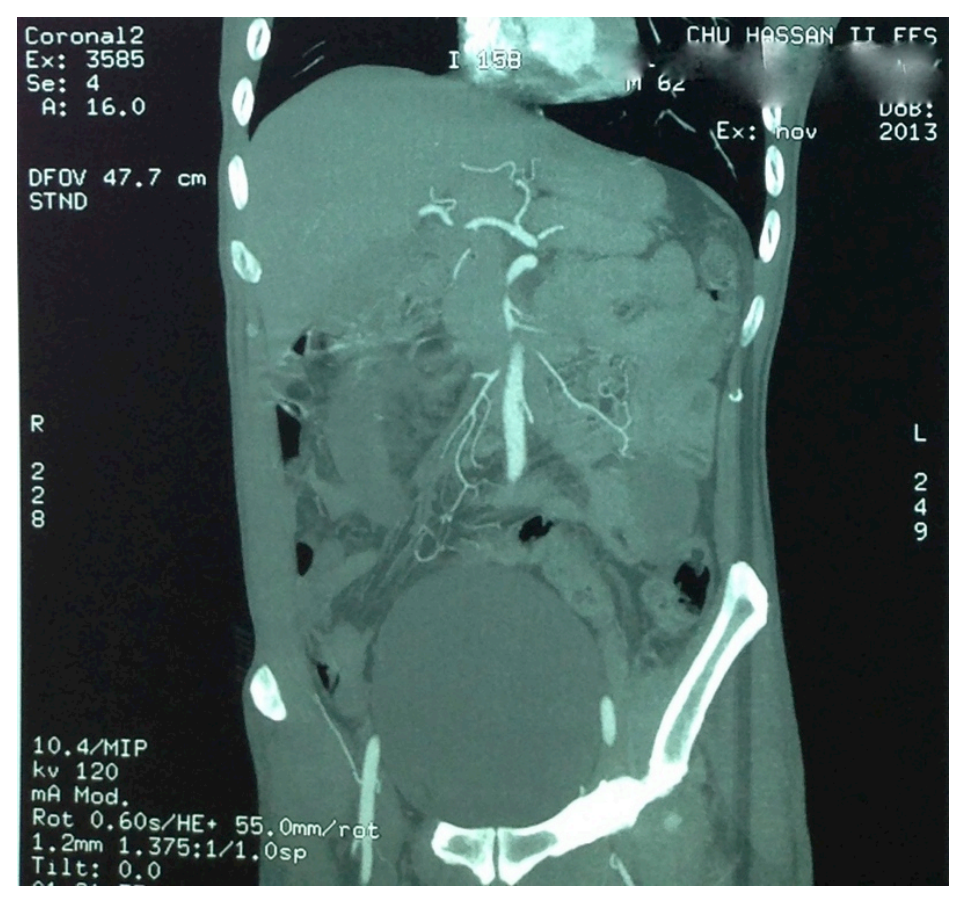

Figure 3. CT scan showing the occlusion of a branch of the SMA by an embolism.

Acute limb ischemia occurs from abrupt interruption of blood flow to an extremity because of either embolic or thrombotic vascular occlusion. The development of profound ischemia is a therapeutic emergency requiring early reperfusion that can lead to limb salvage, whereas delay may result in significant morbidity, including limb loss [8]. Embolic arterial occlusion is the etiology of acute limb ischemia in $15 \%$ of the patients, while arterial thrombosis is the etiology in $85 \%$ of patients and most of them haveatherosclerotic disease. Embolic occlusion brings dramatic limb ischemia and is the most frequent cause of acute limb ischemia [9] while thrombotic occlusion may be less severe and show slower progress thanks to sufficient collateral blood supply. 


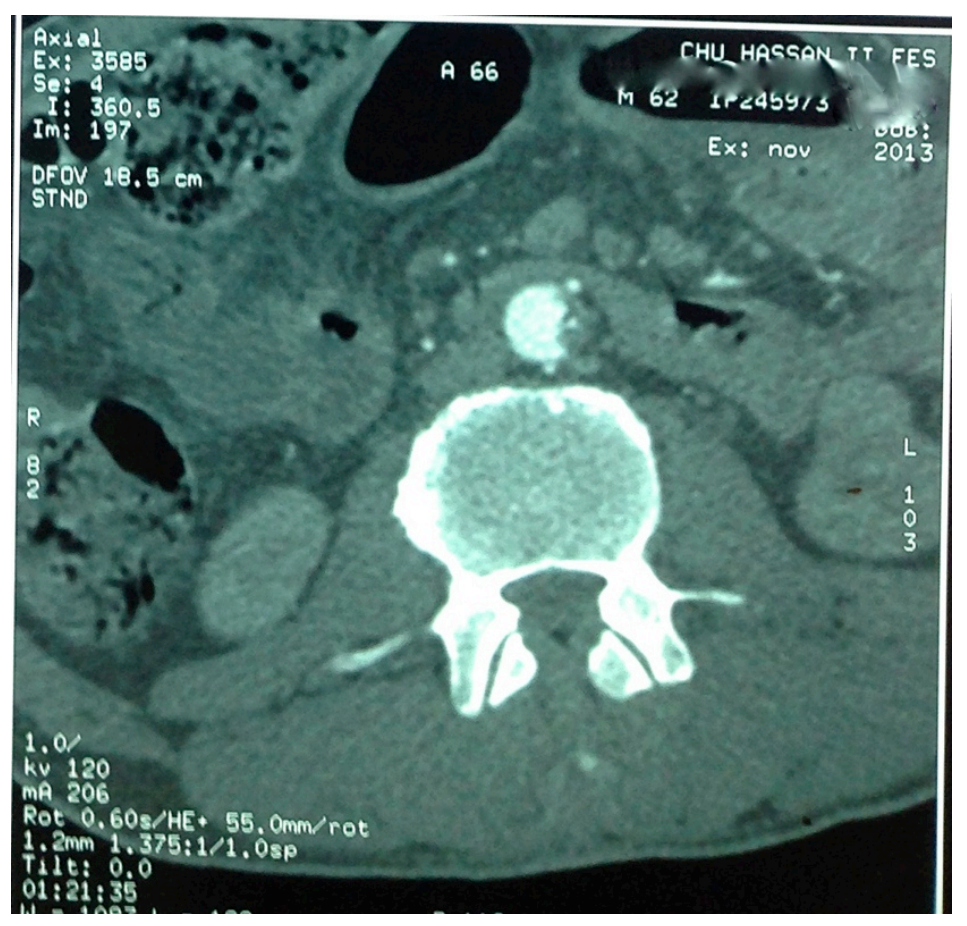

Figure 4. Aortic ulcerated atherosclerotic plaque.

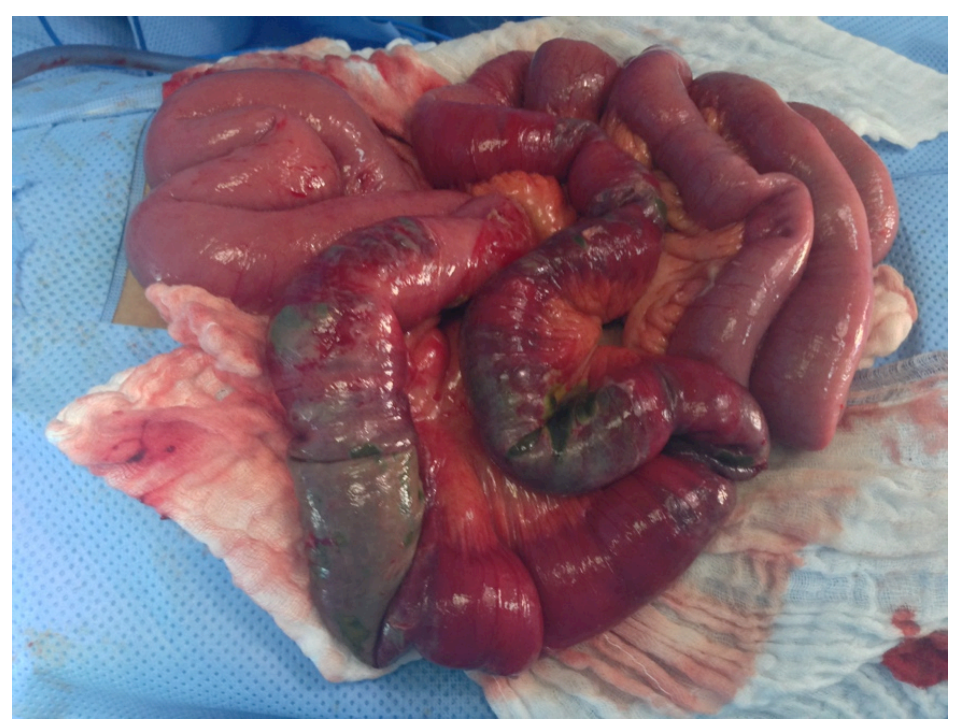

Figure 5. Small bowel infarction.

Embolic episodes following a SMA embolism can occur in the heart, brain, or a lower limb [1]. An embolic episode in a lower limb following an SMA embolism has been previously reported, but it was in the long-term follow-up period (2 - 7 years) [1]. Moreover, a case of upper limb amputation due to a brachial arterial embolism associated with a superior mesenteric arterial embolism was reported in the literature [7]. Because of the severe abdominal symptoms and the shock conditions that followed an emergency enterectomy, the brachial arterial embolism diagnosis was delayed, resulting in amputation of the upper limb. As for our patient, he was diagnosed earlier, allowing limb salvage.

To our knowledge, our patient is the first case described of a concomitant embolism of the SMA and a lower limb embolic episode. He was admitted to the emergency department because of abdominal pain, but on examination, he was found to have bilateral toes ischemia. Because of this vascular background, we suspected a 


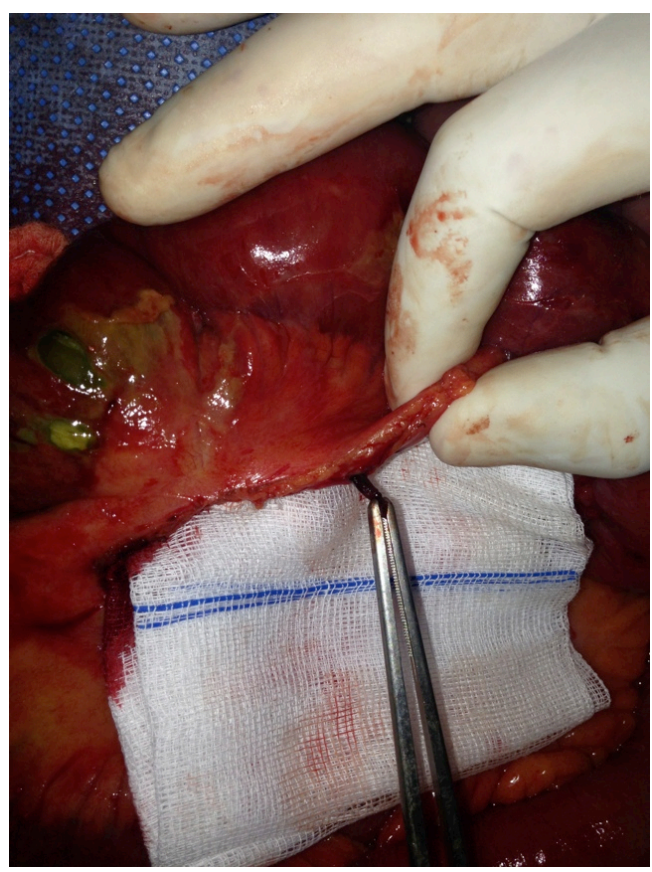

Figure 6. Fresh thrombus in the stump of the resected intestine.

mesenteric ischemia that was confirmed on CT scan. He had an aortic atherosclerotic plaque that was ulcerated andresponsible of both of the SMA embolism that lead to jejunal necrosis, and the embolic arterial occlusion that lead to acute limb ischemia. He was successfully treated by a bowel resection followed by anastomosis, and anticoagulation.

\section{Conclusion}

The possibility of multiple or recurrent thromboembolic events should be considered when treating an AMI due to SMA embolism. It is important to quickly establish the diagnosis, in order to prevent the catastrophic events related to intestinal infarction, and the subsequent development of shower emboli.

\section{Consent}

Written informed consent was obtained from the patient for publication of this Case report and any accompanying images.

\section{Competing Interests}

All authors declare no competing interests.

\section{Author's Contribution}

$\mathrm{AB}$ and $\mathrm{EB}$ participated in writing the case report and revising the draft, all authors participated in the diagnostic and therapeutic management of the patient. All authors read and approved the final manuscript.

\section{References}

[1] Yamaguchi, T., et al. (1999) Local Thrombolytic Therapy for Superior Mesenteric Artery Embolism: Complications and Long-Term Clinical Follow-Up. Radiation Medicine, 17, 27-33.

[2] Lock, G. (2001) Acute Intestinal Ischaemia. Best Practice \& Research Clinical Gastroenterology, 15, 83-98. http://dx.doi.org/10.1053/bega.2000.0157

[3] Bradbury, A.W., Brittenden, J., McBride, K. and Ruckley, C.V. (1995) Mesenteric Ischaemia: A Multidisciplinary 
Approach. British Journal of Surgery, 82, 1446-1459. http://dx.doi.org/10.1002/bjs.1800821105

[4] Sitges-Serra, A., Mas, X., Roqueta, F., Figueras, J. and Sanz, F. (1988) Mesenteric Infarction: An Analysis of 83 Patients with Prognostic Studies in 44 Cases Undergoing a Massive Small-Bowel Resection. British Journal of Surgery, 75, 544-548. http://dx.doi.org/10.1002/bjs.1800750614

[5] Mansour, M.A. (1999) Management of Acute Mesenteric Ischemia. Archives of Surgery, 134, 328-330. http://dx.doi.org/10.1001/archsurg.134.3.328

[6] Haruna, et al. (2012) Acute Mesenteric Ischemia and Duodenal Ulcer Perforation: A Unique Double Pathology. BMC Surgery, 12, 21. http://dx.doi.org/10.1186/1471-2482-12-21

[7] Yamada, et al. (2012) Upper Limb Amputation Due to a Brachial Arterial Embolism Associated with a Superior Mesenteric Arterial Embolism: A Case Report. BMC Research Notes, 5, 372. http://dx.doi.org/10.1186/1756-0500-5-372

[8] Wooyul, P., Mi-Kyeong, O., Jee-Hun, K., Ha-Gyoung, K. and Sang-Sig, C. (2011) Paroxysmal Atrial Fibrillation Presenting as Acute Lower Limb Ischemia. Korean Journal of Family Medicine, 32, 423-427. http://dx.doi.org/10.4082/kjfm.2011.32.7.423

[9] Walker, T.G. (2009) Acute Limb Ischemia. Techniques in Vascular and Interventional Radiology, 12, 117-129. http://dx.doi.org/10.1053/j.tvir.2009.08.005 